\title{
Morphology and Photophysical Properties of Light-Emitting Electrospun Nanofibers Prepared from Poly(fluorene) Derivative/ PMMA Blends
}

\author{
Chi-Ching Kuo, ${ }^{\dagger}$ Chia-Hung Lin,,$*$ and Wen-Chang Chen $*,+$, \\ Institute of Polymer Science and Engineering, National Taiwan University, Taipei 106, Taiwan, and \\ Department of Chemical Engineering, National Taiwan University, Taipei 106, Taiwan
}

Received May 25, 2007; Revised Manuscript Received July 1, 2007

\begin{abstract}
Light-emitting electrospun (ES) nanofibers with diameters of 250-750 nm were successfully prepared through the binary blends of polyfluorene derivative/poly(methyl methacrylate) (PMMA) using a singlecapillary spinneret. The studied poly(fluorene)s included poly(9,9-dioctylfluoreny-2,7-diyl) (PFO), poly[2,7-(9,9dihexylfluorene)-alt-5,8-quinoxaline] (PFQ), poly[2,7-(9,9-dihexylfluorene)-alt-4,7-(2,1,3-benzothiadiazole)] (PFBT), and poly[2,7-(9,9-dihexylfluorene)-alt-5,7-(thieno[3,4-b]pyrazine)] (PFTP). The transmission electron microscopy (TEM) studies showed that uncontinuous fiberlike structure was obtained at the low PFO/PMMA blend ratio but became a core-shell structure at a high PFO blend ratio. The poorer solubility of PFO in chloroform than that of PMMA probably forced it to be solidified first as the fiber core. Besides, a porous surface structure on the PFO/PMMA blend fibers was observed due to the rapid evaporation of the chloroform solvent. The PFO aggregation domain in the ES fibers was much smaller than that in the spin-coated films and led to higher photoluminescence efficiency. Uniform ES fibers produced from the binary blends of PFO/PMMA, PFQ/PMMA, PFBT/PMMA, and PFTP/PMMA exhibited the following luminescence characteristics (peak maximum (nm); color): 443, blue; 483, green; 539, yellow; 628, red. The present study demonstrates that full color light-emitting ES nanofibers could be produced from the binary blends of polyfluorene derivative/PMMA.
\end{abstract}

\section{Introduction}

Conjugated polymers have been extensively studied for various electronic and optoelectronic devices, such as electroluminescence displays, photovoltaic cells, and field effect transistors. ${ }^{1-5}$ Oligo- and polyfluorene as well as their derivatives are excellent candidates for optoelectronic applications because they exhibit high thermal/chemical stability, excellent fluorescence quantum yields, and substantial charge carrier mobility. The photophysical properties of polyfluorene could be tuned through the approaches of copolymer, blend, side chain modification, or end-capping and result in an enhancement of device characteristics. ${ }^{6,7}$ However, most of the above studies are based on the thin film devices. The morphology and properties of polyfluorene based nanofibers have not been fully explored yet.

Electrospinning (ES) has emerged as a new technique to produce various functional nanofibers ${ }^{8,9}$ because of its advantages of low cost, flexible morphology tuning, and a highthroughput continuous production process. However, only few ES nanofibers based on conjugated polymers were reported because of the limitations on molecular weight or solvents..$^{10-12}$ Highly conductive ES nanofibers prepared from acid-doped polyaniline/ polystyrene blends were reported by MacDiarmid and his co-workers. ${ }^{10 \mathrm{a}, \mathrm{b}}$ Tunable luminescence characteristics were demonstrated on the ES nanofibers of poly[2-methoxy5-(2-ethylhexoxy)-1,4-phenylene] (MEH-PPV)/silica ${ }^{11 a}$ and poly(aryleneethynylene)s. ${ }^{11 g}$ Recently, the morphology and

* Author to whom all correspondence should be addressed. Telephone: 886-2-23628398. Fax: 886-2-23623040. E-mail: chenwc@ntu.edu.tw.

$\dagger$ Institute of Polymer Science and Engineering, National Taiwan University.

$\doteqdot$ Department of Chemical Engineering, National Taiwan University.
Chart 1. Chemical Structures of the Studied Polyfluorene Derivatives: (a) PFO, (b) PFQ, (c) PFBT and (d) PFTP

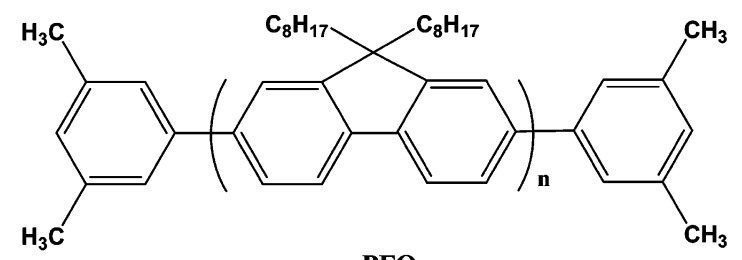

PFO

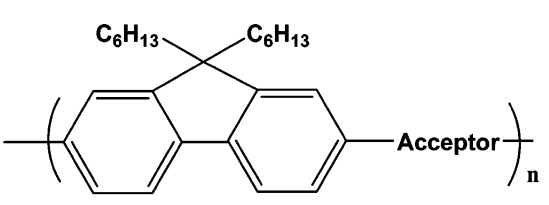

Acceptor $=$

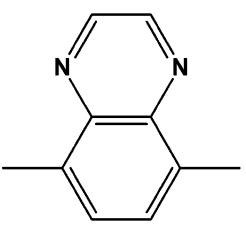

PFQ

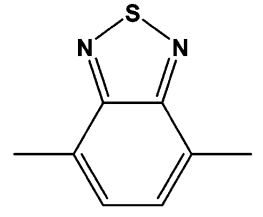

PFBT

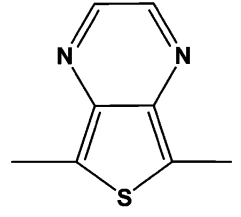

PFTP photophysical properties of ES nanofibers based on MEH-PPV with poly(3-hexylthiopehen) (PHT) and with poly(9,9-dioctylfluoreny-2,7-diyl) (PFO) were studied for field effect transistor devices. ${ }^{12 \mathrm{a}, \mathrm{b}}$ The $\mathrm{MEH}-\mathrm{PPV} / \mathrm{PFO}$ blend nanofibers showed a core-shell structure but without significant energy transfer, which was quite different from the blend thin films. ${ }^{12 b}$ It suggests the morphology and photophysical properties of ES nanofibers might be very different from those of thin films. The 
(a)

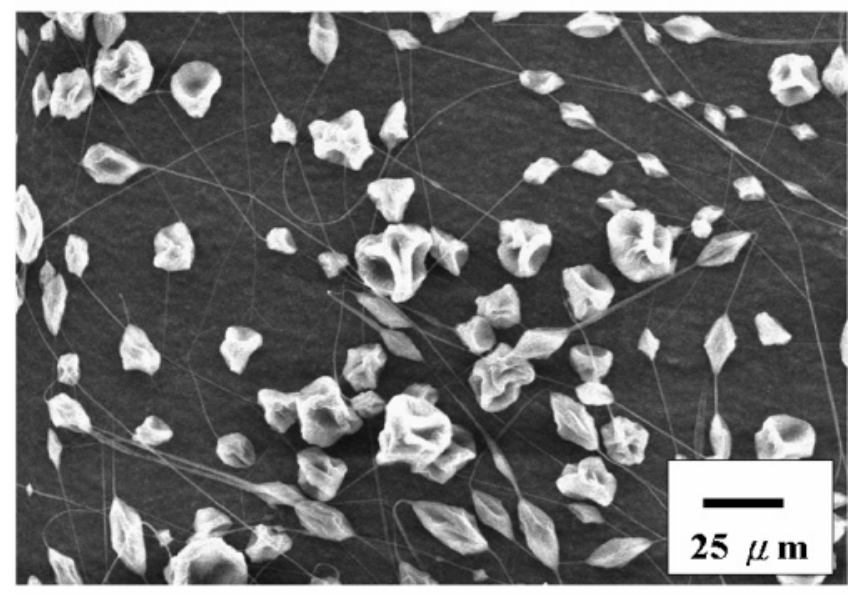

(b)

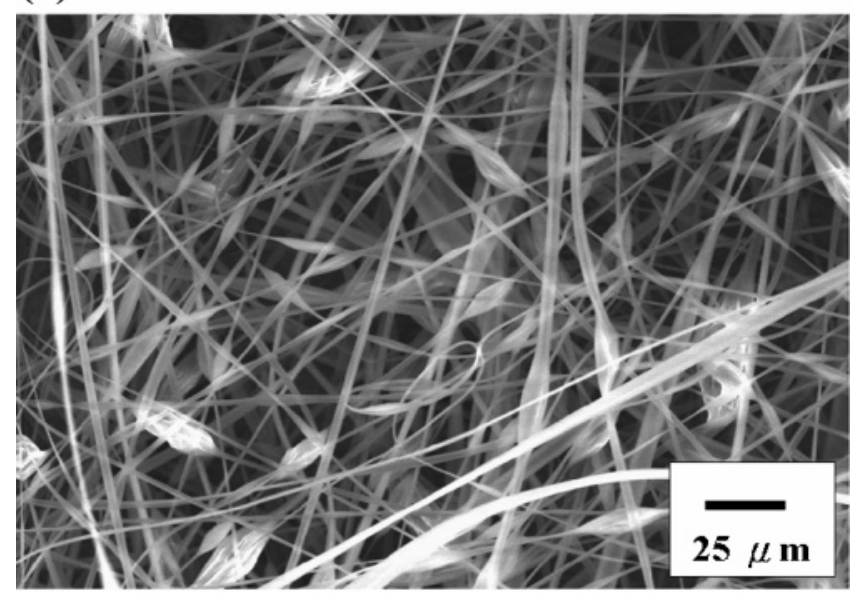

(c)

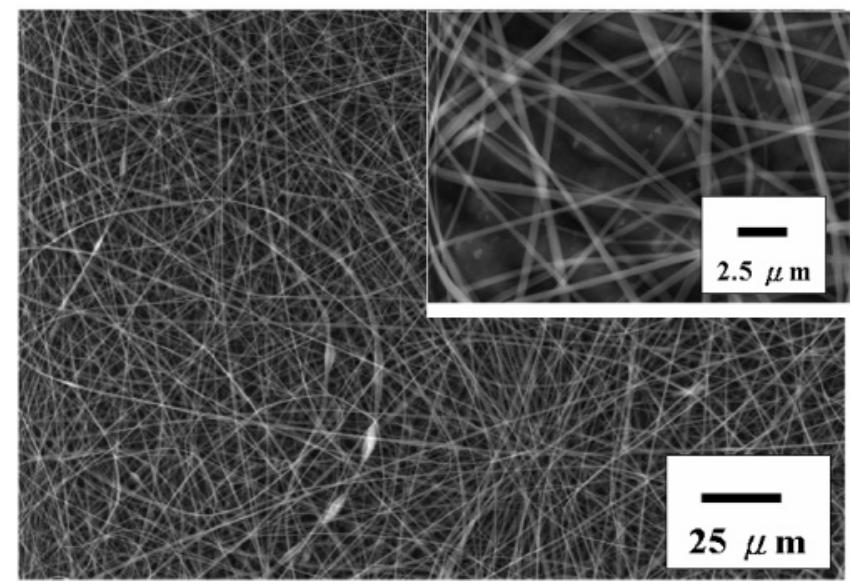

Figure 1. FE-SEM images of $10 \mathrm{wt} \%$ PFO/PMMA blend ES nanofibers with different concentrations of BTEAC (wt \%): (a) 0; (b) 0.5 ; (c) 3 (with respect to polymer).

polyfluorene/nonconjugated polymer blend films were shown to reduce the PF aggregation and thus enhancement of luminescence efficiency was demonstrated..$^{7 a-d}$ However, to the best of our knowledge, the morphology and photophysical properties of polyfluorene/nonconjugated polymer ES fibers have not been reported yet. Besides, tuning the luminescence color through the visible range and quantum efficiency in such ES fibers also needs to be explored further. (a)

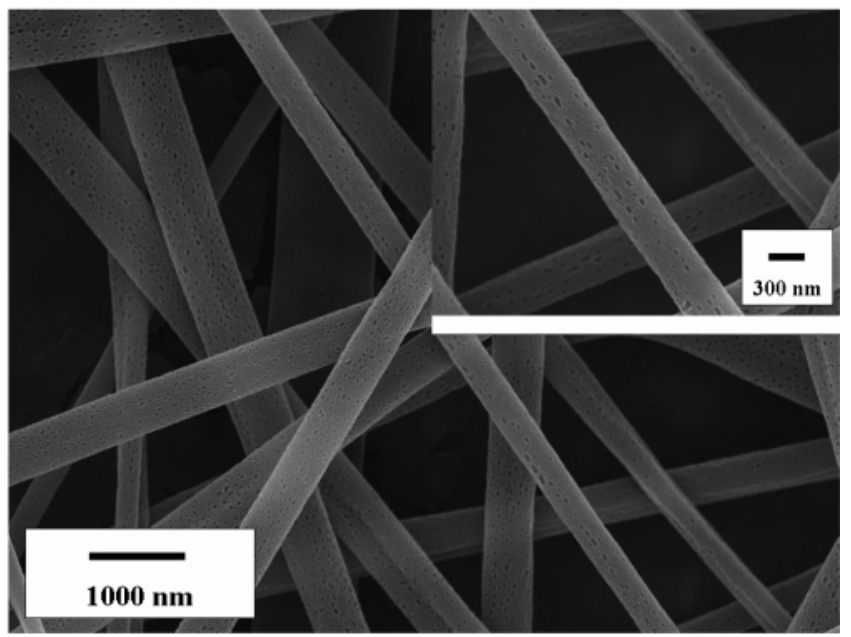

(b)

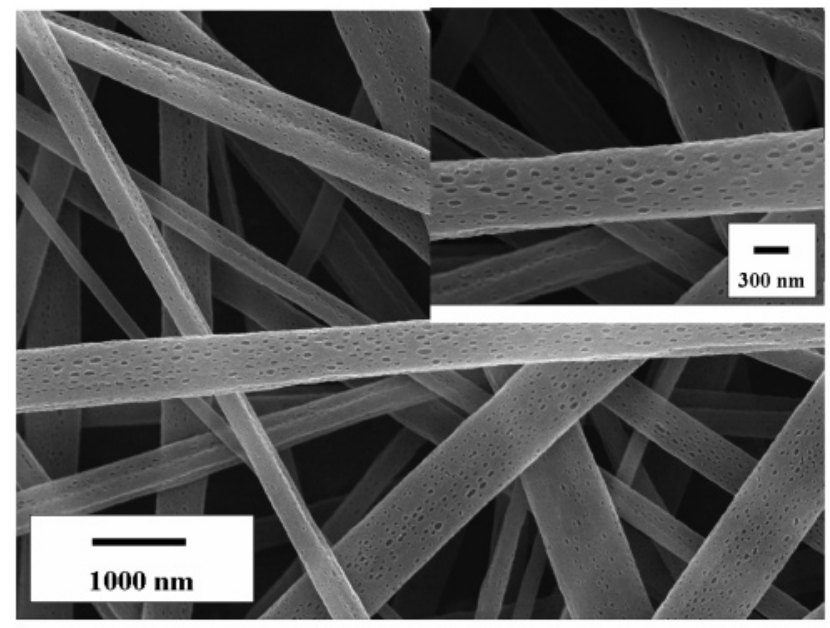

Figure 2. FE-SEM images of PFO/PMMA blend ES nanofibers with (a) 10 and (b) $50 \mathrm{wt} \%$ of PFO.

In this study, we report the morphology and photophysical properties of ES nanofibers based on the binary blends of polyfluorene derivative/ poly(methyl methacrylate)(PMMA) blends using a single-capillary spinneret. The studied polyfluorene derivatives included poly(9,9-dioctylfluoreny-2,7-diyl) (PFO), poly [2,7-(9,9-dihexylfluorene)-alt-5,8-quinoxaline] (PFQ), poly[2,7-(9,9-dihexyl- fluorene)-alt-4,7-(2,1,3-benzothiadiazole)] (PFBT), and poly[2,7-(9,9-dihexyl- fluorene)-alt-5,7-(thieno[3,4b]pyrazine)] (PFTP). The chemical structures of the studied polymers are shown in Chart 1. The morphology and diameter of the prepared ES nanofibers were varied through different spinning conditions and then characterized by field-emission scanning electron microscope (FE-SEM), transmission electron microscope (TEM), and laser confocal microscope (Confocal). The photophysical properties of the ES fibers were investigated by optical absorption and steady-state photoluminescence (PL). Our results demonstrated the PFO/PMMA ES nanofibers could form a core-shell structure at a high PFO content and had higher luminescence efficiencies than those of spin-coated thin films. Beside, full color luminescence ES fibers were produced by varying the polyfluorne derivative/PMMA blend. 
Table 1. Compositions and Photophysical Properties of the Electrospun Fibers and Spin-Coated Films

\begin{tabular}{|c|c|c|c|c|c|}
\hline & & composition (wt $\%)^{a}$ & $\left(\lambda_{\max }^{\mathrm{abs}}\right)(\mathrm{nm})$ & $\left(\lambda_{\max }^{\mathrm{PL}}\right)(\mathrm{nm})$ & PL efficiency (\%) \\
\hline \multirow[t]{8}{*}{ electrospun fibers } & PFO/PMMA & 1 & 397 & 421,442 & 75.5 \\
\hline & PFO/PMMA & 5 & 398 & 425,443 & 54.7 \\
\hline & PFO/PMMA & 10 & 399 & 426,444 & 54.0 \\
\hline & PFO/PMMA & 30 & 403,436 & $426,444,446$ & 52.4 \\
\hline & PFO/PMMA & 50 & 406,436 & $426,444,466,501$ & 44.6 \\
\hline & PFQ/PMMA & 5 & 324,406 & 483 & 33.3 \\
\hline & PFBT/PMMA & 5 & 335,458 & 539 & 24.5 \\
\hline & PFTP/PMMA & 5 & $314,356,531$ & $437,628,658$ & 3.9 \\
\hline \multirow[t]{8}{*}{ spin-coated films } & PFO/PMMA & 1 & 397,433 & $438,463,495$ & 58.2 \\
\hline & PFO/PMMA & 5 & 397,433 & $439,463,495$ & 50.2 \\
\hline & PFO/PMMA & 10 & 397,433 & $440,463,495$ & 48.8 \\
\hline & PFO/PMMA & 30 & 398,433 & $441,465,498$ & 45.4 \\
\hline & PFO/PMMA & 50 & 403,433 & $441,469,500,533$ & 41.1 \\
\hline & PFQ/PMMA & 5 & 326,409 & 477 & 11.2 \\
\hline & PFBT/PMMA & 5 & 324,468 & 536 & 15.4 \\
\hline & PFTP/PMMA & 5 & $314,364,560$ & 631,661 & 3.6 \\
\hline
\end{tabular}

${ }^{a}$ The weight ratio of polyfluorene derivative/ PMMA

\section{Experimental Section}

Materials. The PMMA $\left(M_{\mathrm{w}} \sim 350000\right)$ was purchased from Aldrich. PFO $\left(M_{\mathrm{w}} \sim 101000\right)$ was obtained from American Dye Source, Inc. Poly[2,7-(9,9-dihexylfluorene)-alt-5,8- quinoxaline] $\left(\mathrm{PFQ}, M_{\mathrm{w}}=35530, \mathrm{PDI}=1.87\right)$, poly[2,7-(9,9-dihexyl-fluorene) -alt-4,7-(2,1,3-benzothiadiazole)] (PFBT, $M_{\mathrm{w}}=39900$, PDI $=$

(a)

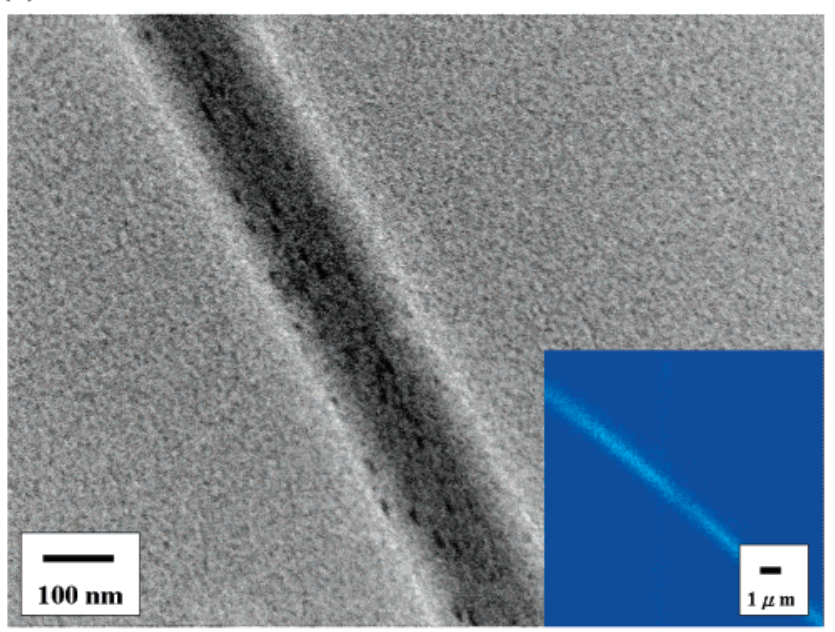

(b)

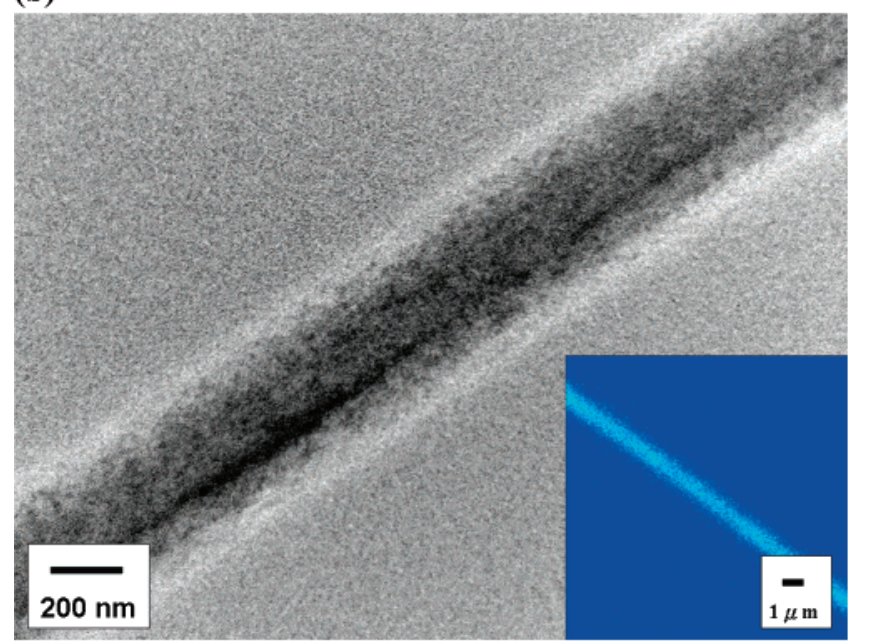

2.10), and poly[2,7-(9,9-dihexylfluorene)-alt-5,7-(thieno[3,4-b]pyrazine) $]\left(\right.$ PFTP, $\left.M_{\mathrm{w}}=13230, \mathrm{PDI}=1.47\right)$ were prepared according to our previous report. ${ }^{6 \mathrm{~d}}$ High purity chloroform (HPLC grade, TEDIA) and benzyl triethylammonium chloride (BTEAC, Aldrich) were used as received.

Electrospinning Setup. The PFO/PMMA blend with the concentration of $100 \mathrm{mg} / \mathrm{mL}$ in chloroform and the benzyl triethylam-

(c)

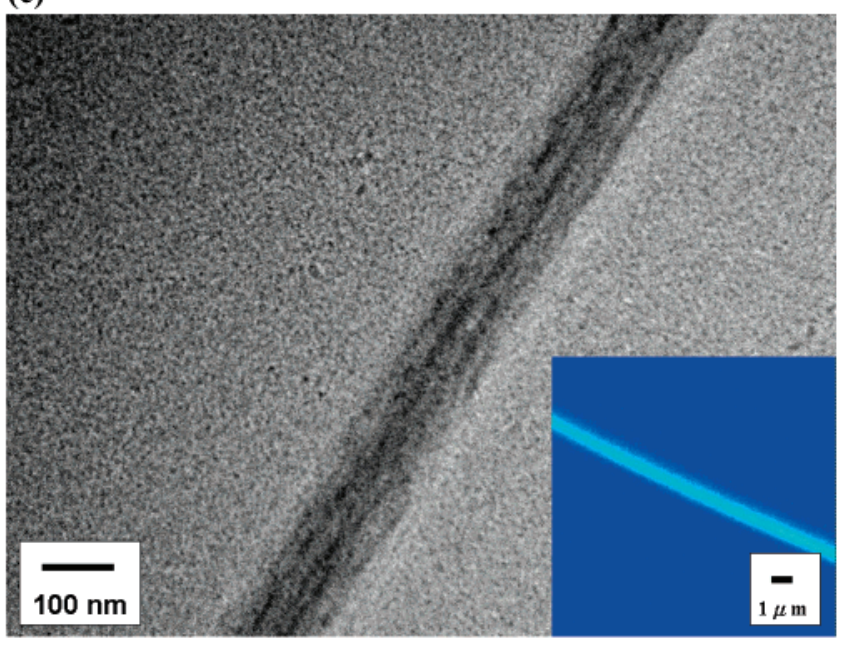

(d)

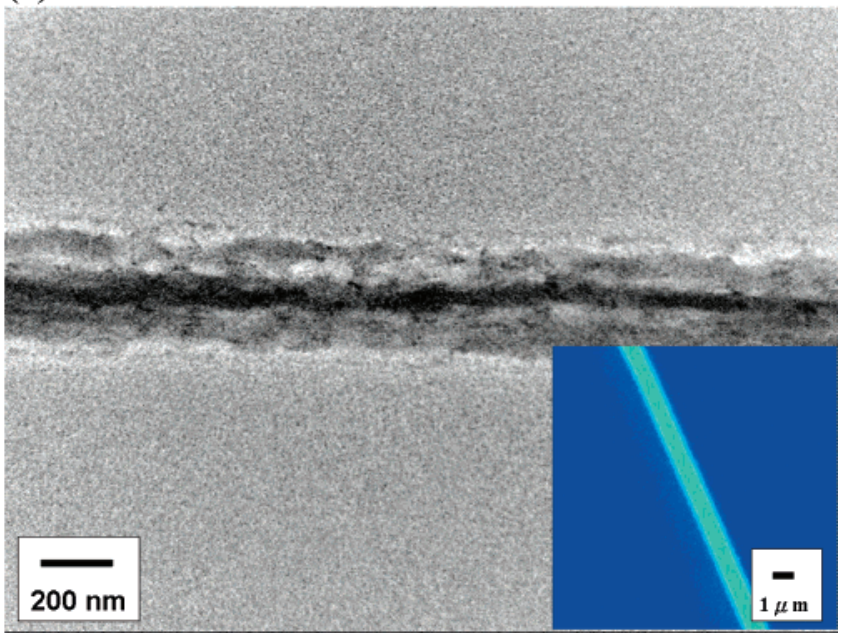

Figure 3. TEM images of ES nanofibers prepared from (a) 1, (b) 10, (c) 30, and (d) $50 \mathrm{wt} \%$ PFO blends. The insert figure illustrates the laser confocal microscope images of the above ES fibers. 
(a)

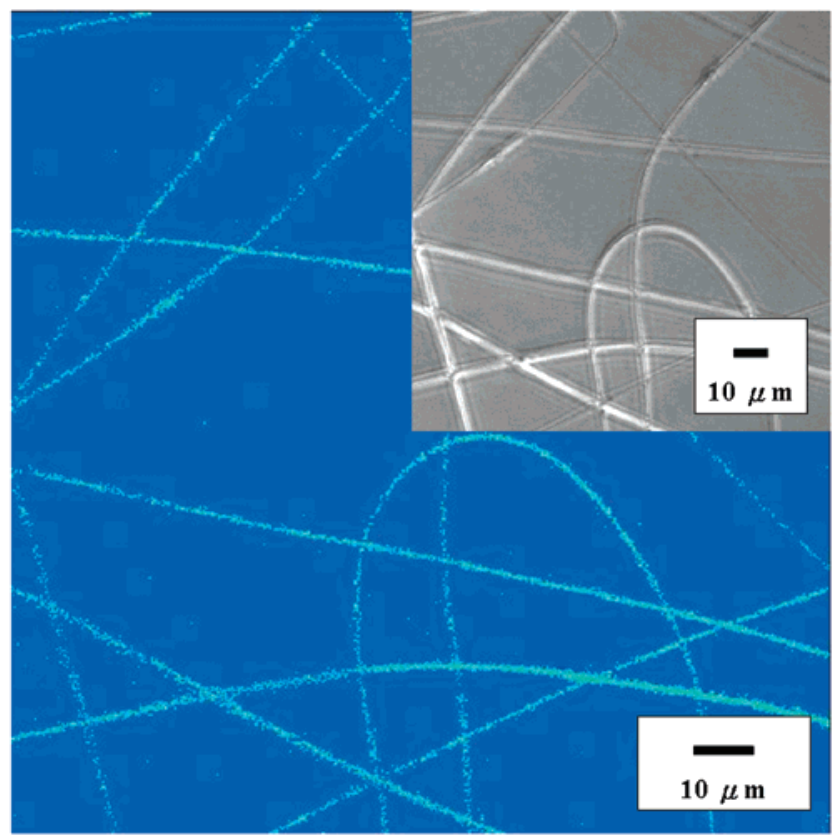

(b)

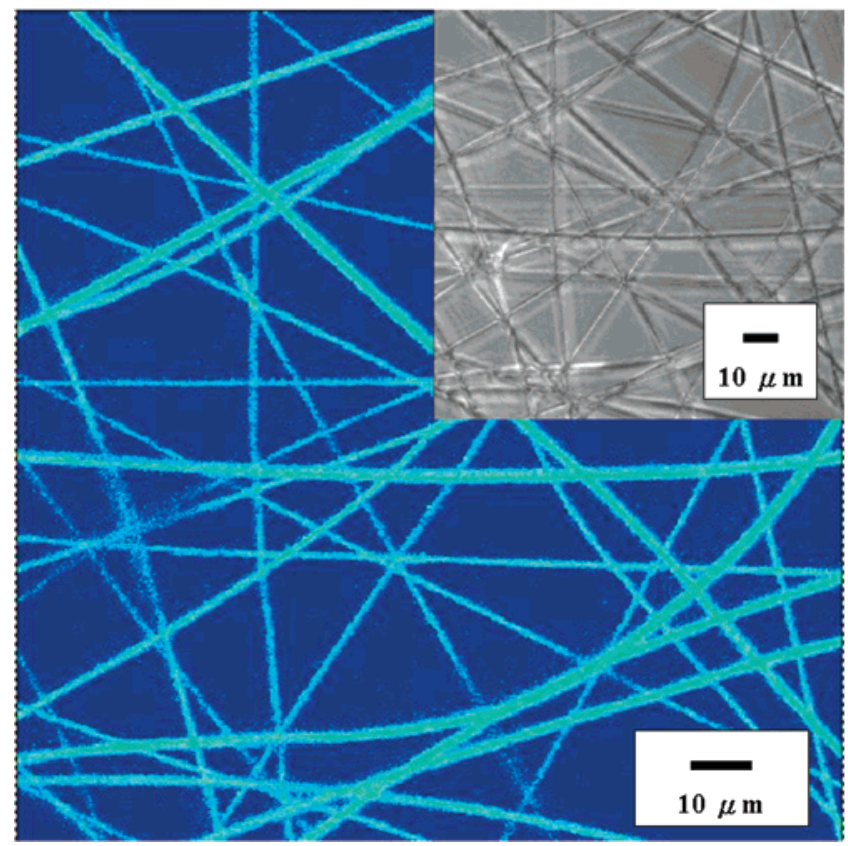

(c)

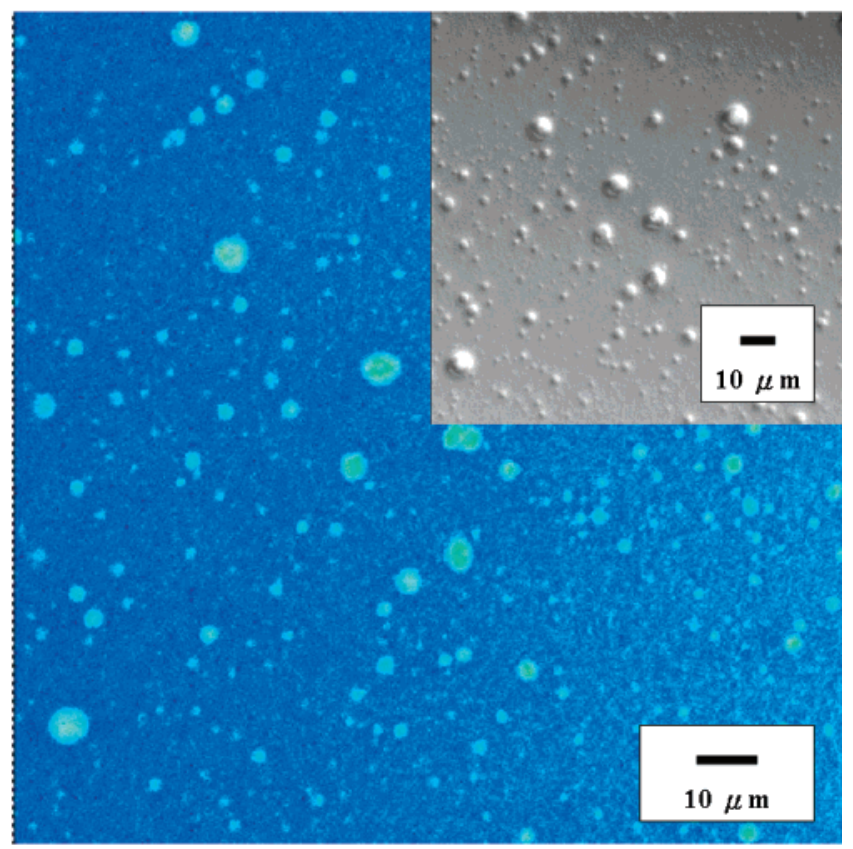

(d)

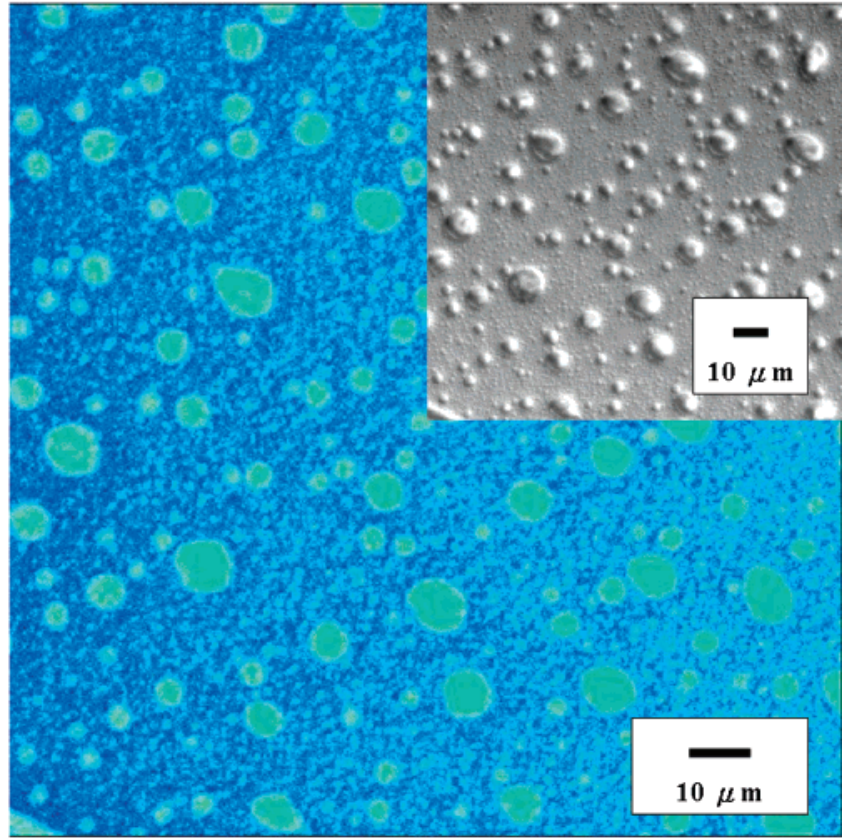

Figure 4. Laser confocal microscope images of (a) 10 and (b) $30 \mathrm{wt} \%$ PFO ES fibers and the corresponding spin-coated films (c and d). The inset shows the optical images of the above ES fibers and spin-coated films.

monium chloride (BTEAC, a soluble organic salt) of $0-3 \mathrm{wt} \%$ (with respect to the polymer) were used to produce the ES fibers. Note that BTEAC was soluble in chloroform and easily to be removed from the resulting ES fibers by a methanol treatment. It was found out that the salt concentration of $3 \mathrm{wt} \%$ BTEAC was able to produce uniform PFO/PMMA fibers (see the results and discussion) and used to prepare the ES fibers in the present study. The polymer blend compositions of PFO, PFQ, PFBT, and PFTP with PMMA are listed in Table 1 . The PFO blend was varied from 1 to $50 \mathrm{wt} \%$ while others were fixed at $5 \mathrm{wt} \%$ with respect to PMMA.

The ES fibers were prepared using a single-capillary spinneret. First, the solution was fed into the syringe pumps (KD Scientific model 100) connected to the metallic needle, with the feed rate of $2 \mathrm{~mL} / \mathrm{h}$. The metallic needle was connected to a high-voltage power supply (chargemaster CH30P SIMCO), and a piece of aluminum foil or silicon wafer was placed $15 \mathrm{~cm}$ below the tip of the needle to collect the nanofibers. The spinning voltage was set at $20 \mathrm{kV}$. All experiments were carried out at room temperature.

For the comparison with morphology and properties of the ES fibers, thin films of the polyfluorene derivative/PMMA blends were spin-coated from chloroformsolutions at a spin rate of $1500 \mathrm{rpm}$ for $60 \mathrm{~s}$.

Morphology of Electrospun Fibers and Thin Films. The morphologies of ES fiber and spin-coated films prepared were characterized by field-emission scanning electron microscope (FE- 


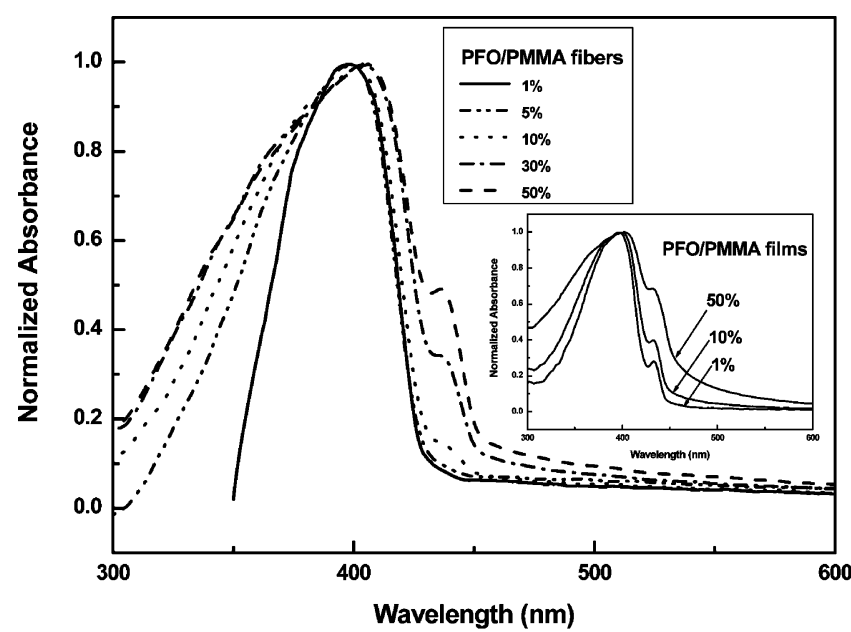

Figure 5. UV-vis absorption spectra of the 1-50 wt \% PFO blend ES fibers and those of the corresponding blend spin-coated films are shown in the inset figure.
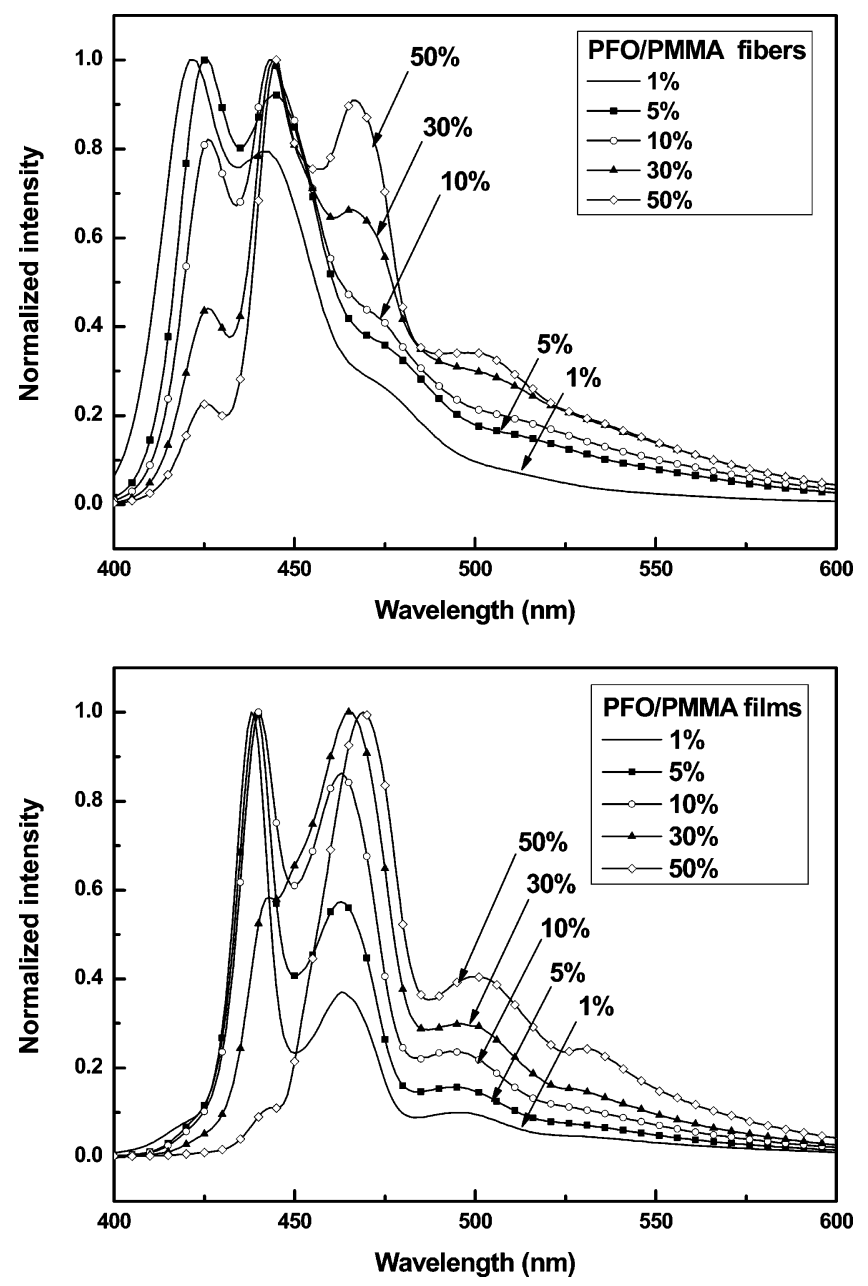

Figure 6. Photoluminescence emission spectra of (a) PFO/PMMA blend ES nanofibers and (b) spin-coated thin films.

SEM) (JEOL JSM-6330F). Those images were taken using a microscope operated at an accelerating voltage of $15 \mathrm{kV}$. Before imaging, the samples were sputtered with $\mathrm{Au} / \mathrm{Pt}$. TEM images were taken using a Transmission Electron microscope (JOEL JEM-1230) operated at $100 \mathrm{keV}$. Fluorescence optical microscope images were taken using a two-photon laser confocal microscope (Leica LCS $\mathrm{SP} 2$ ).

Photophysical Properties. UV-visible absorption and photoluminescence (PL) spectra were recorded on UV-visible spectrophotometer (Hitachi U-4100) and Fluorolog-3 spectrofluorometer
(Jobin Yvon), respectively. The PL efficiencies of ES fibers and spin-coated films onto quartz substrates were measured using a Fluorolog 3 in combination with an integration sphere with 380 $\mathrm{nm}$ excitation, as described in our previous study. ${ }^{6 \mathrm{~d}}$

\section{Results and Discussion}

The BTEAC salt concentration was optimized first to produce uniform ES fibers. Figure $1 \mathrm{a}-\mathrm{c}$ shows the FE-SEM images of 10 wt \% PFO/PMMA blend ES fibers prepared at solution concentrations of $100 \mathrm{mg} / \mathrm{mL}$ with the BTEAC concentrations of $0,0.5$, and 3 wt $\%$ (with respect to polymer), respectively. Without the BTEAC salt, a bead-fiber structure is observed at Figure 1a, due to the low solution viscosity. Such a bead-forming structure was resolved by adding the BTEAC salt since it could enhance the surface tension and conductivity. ${ }^{9}$ The bead density is largely reduced and becomes insignificantly by adding 0.5 wt $\%$ (Figure 1b) and 3 wt \% (Figure 1c) of BTEAC, respectively. The continuous PFO/PMMA ES fibers with the diameters ranging from 250 to $750 \mathrm{~nm}$ are shown in Figure 1c. Hence, the following experimental conditions were used to prepare all of the following ES fibers for discussion: polymer solution concentration of $100 \mathrm{mg} / \mathrm{mL}$ in chloroform with $3 \mathrm{wt}$ $\%$ (with respect to polymer) of BTEAC.

The FE-SEM images of 1,30 , and 50 wt \% PFO ES nanofibers shown in Figure S1 (Supporting Information) shows that the fiber diameters are in the range of $250-750 \mathrm{~nm}$. The similar fiber diameter in different PFO blend ratio is as expected since the fixed concentration of the ES solution and the much larger molecular weight of PMMA than that of PFO. However, the numbers of beads were also enhanced with increasing PFO blend ratio, as shown in Figure S1, parts b and c. It might result from the more rigid structure of PFO as compared with that of PMMA and increasing PFO aggregation at a high blend ratio.

Parts $a$ and $b$ of Figure 2 show the FE-SEM images of the 10 and $50 \mathrm{wt} \%$ PFO/PMMA blend ES fibers, respectively. The insert figures show the enlarged FE-SEM images of the corresponding ES fibers. A porous surface structure with the pore size of $50-150 \mathrm{~nm}$ is exhibited in both blend ES fibers. Besides, the strong stretching force associated with electrospinning induces orientation of these pores along the axis of a fiber. The 1 and 30 wt \% PFO ES fibers also exhibit a porous surface structure, as shown in Figure S2, parts a and b, of the Supporting Information. The porous structure is probably resulted from the rapid evaporation of the chloroform solvent and a subsequent rapid solidification during the ES process, similar to that observed in the literature. ${ }^{11 \mathrm{f}}$ These porous ES fibers might increase surface-to-volume ratios and thus have potential applications in photonics and sensors. ${ }^{11 \mathrm{~b}, \mathrm{c}}$

Figure 3 shows that TEM images of ES nanofibers prepared from (a) 1, (b) 10, (c) 30, and (d) 50 wt \% PFO blends, respectively. The insert figures show the laser confocal microscopy images of the corresponding ES fibers. PFO is a conjugated polymer with a significant $\pi$-electron delocalization and may have a higher electronic density than PMMA. Thus, the dark fiber-like structure in the TEM image is assigned to the PFO domains. As shown in Figure 3a, PFO phase-separated fiberlike domains with dimension size of $1-5 \mathrm{~nm}$ in the fiber center is observed in the image of the $1 \mathrm{wt} \%$ PFO ES fibers. Such fiber-like domains are increased as $\mathrm{PF}$ blend ratios are enhanced from 10, 30, and 50\% PFO blend ratios; 5-10 (Figure 3b), 1020 (Figure 3c), and 40-60 nm (Figure 3d), respectively. Besides, a core-shell structure with the aligned fiber-like structure is observed in the $50 \mathrm{wt} \%$ PFO blend ES fibers of Figure $3 \mathrm{~d}$. It suggests that the uncontinuous fiber-like structure 
(a)

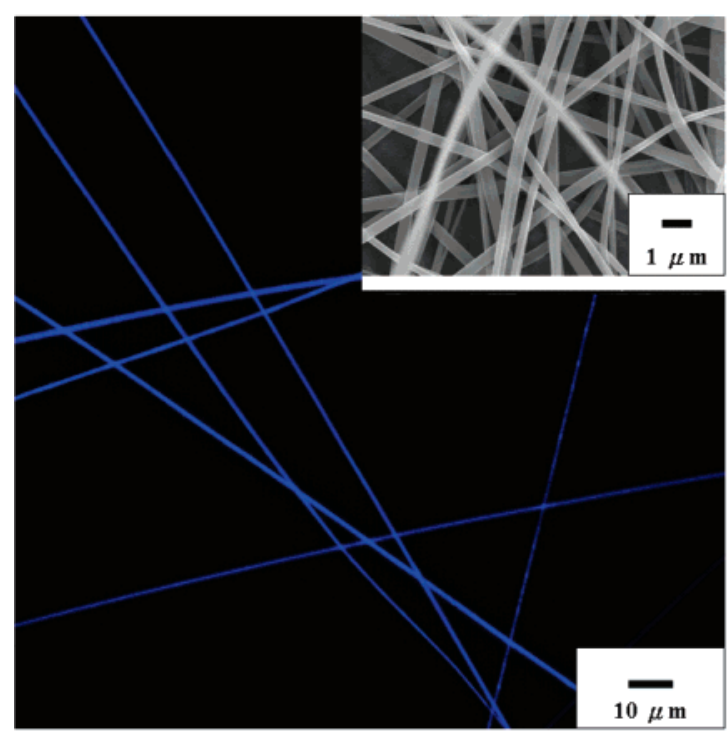

(c)

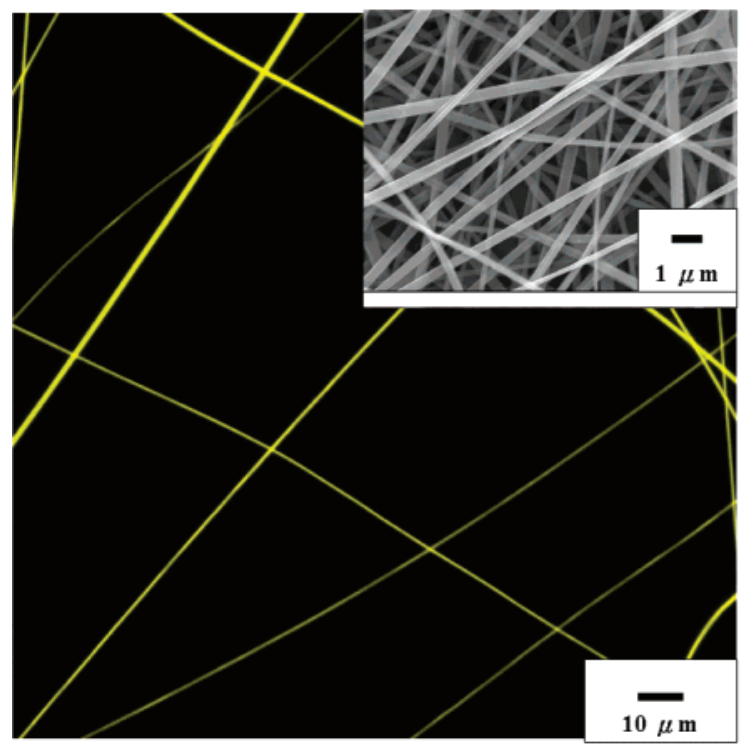

(b)

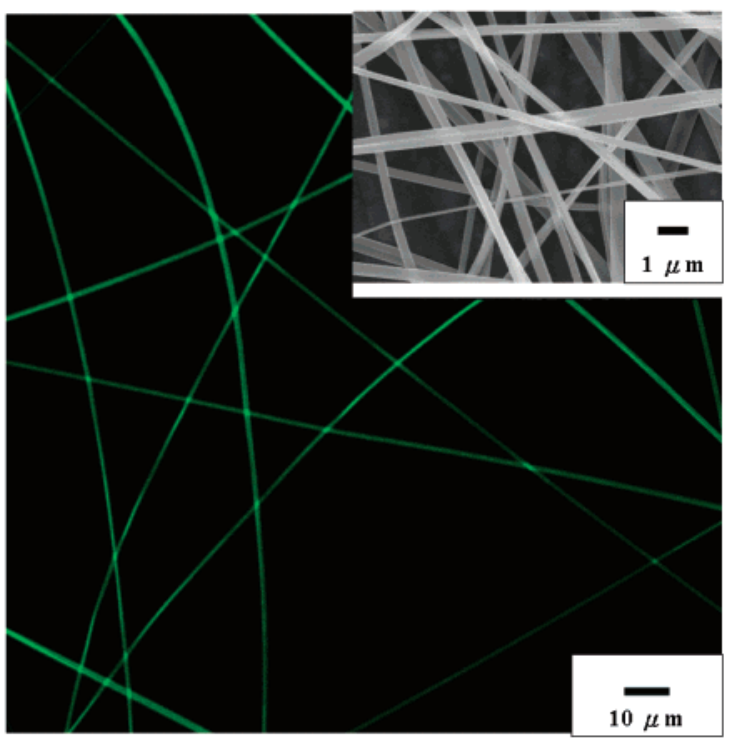

(d)

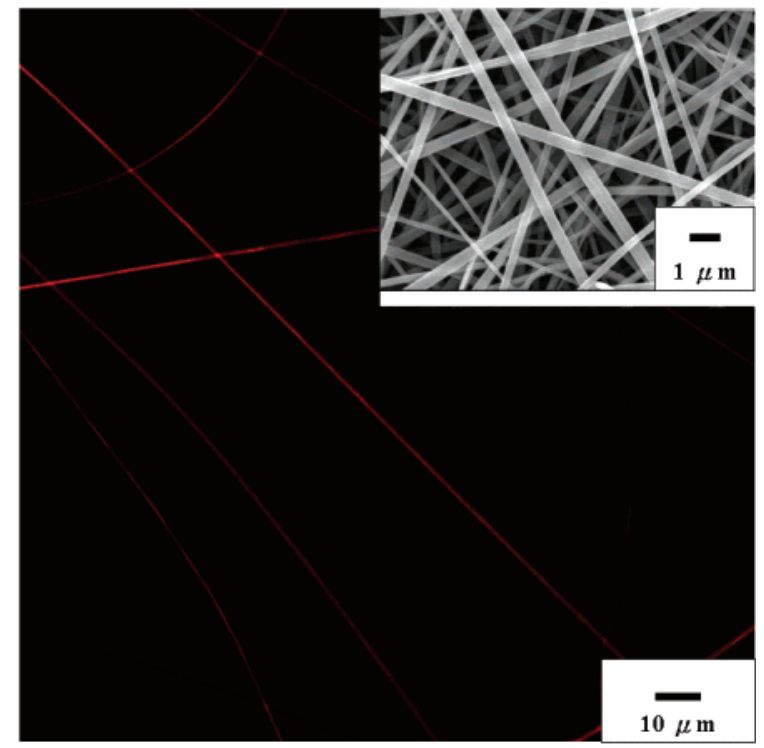

Figure 7. Laser confocal microscopy images of 5 wt \% polyfluorene derivative/ PMMA blend ES nanofibers with (a) PFO; (b) PFQ; (c) PFBT; (d) PFTP

is obtained at the low PFO blend ratio but becomes continuous fiber-like structure at a high $\mathrm{PFO}$ blend ratio. The rigid structure of the PFO could have a poorer solubility in chloroform than PMMA and thus solidify quicker than PMMA during the ES process. Thus, PFO is formed at the fiber core and subsequently PMMA as the fiber shell. Moreover, many studies have shown that the strong stretching forces associated with electrospinning may induce orientation of polymer chains along the long axis of fiber. ${ }^{13}$ It is expected that such aligned nanofibers of conjugated polymers may exhibit unique properties such as high charge-carrier mobility ${ }^{12 \mathrm{a}, \mathrm{b}}$ or polarzed photoluminescence. ${ }^{11 \mathrm{f}, 14}$

Figure 4 shows the laser confocal and optical (insert figure) images of the 10 and $30 \mathrm{wt} \%$ PFO blend ES fibers and spincoated films, respectively. Parts a and b of Figure 4 show uniform blue ES fibers and smooth optical images in the inset figures. However, large aggregated domains with the sizes 100 $\mathrm{nm} \sim 50 \mu \mathrm{m}$ are shown in the blend films of Figure 4, parts $\mathrm{c}$ and $\mathrm{d}$. The numbers and domain sizes of aggregates of Figure $4 \mathrm{~d}$ are also much larger than those of Figure $4 \mathrm{c}$, which suggests that they result from the PFO. The confinement of the liquid jets during the ES process probably results in smaller phaseseparated domains than those of the spin-coated films, which is similar to that reported in the literature. ${ }^{12 b}$ Furthermore, the ES process may produce more extended PFO chain conformation along the fiber axis and thus reduce the aggregation of PFO in comparison with the spin-coated films. Such difference on the aggregated domain would result in a significant variation on the photophysical properties, as discussed below.

Photophysical Properties. Figure 5 shows the UV-vis absorption spectra of the PFO/PMMA ES fibers and those of the corresponding spin-coated films are shown in the inset figure. As shown in the figure, only single absorption maximum $\left(\lambda_{\max }{ }^{a b s}\right)$ around $397 \mathrm{~nm}$ is observed in the absorption spectra of 1-10 wt \% PFO blend ratio but a side absorption band with 
$\lambda_{\max }{ }^{\text {abs }}$ at $436 \mathrm{~nm}$ at a higher PFO content. However, the absorption peak maxima around 397-403 and $433 \mathrm{~nm}$ are shown in all of the blend ratios for the blend films. Since the optical absorption maximum of the PMMA is below $250 \mathrm{~nm}$, the absorption band at $397 \mathrm{~nm}$ is attributed to the PFO, which is also close to that reported for the PFO/PMMA films. ${ }^{7 \mathrm{c}}$ Although there is insignificant difference on the absorption band around $397 \mathrm{~nm}$, the intensity of the side band is different on the high PFO blend ratio between the ES fibers and thin films. The side band around $436 \mathrm{~nm}$ is attributed to the PFO aggregates with an ordered intrachain conformation and a more delocalized ground-state structure. ${ }^{7 \mathrm{a}}$ The evolution of the side band at 433 or $436 \mathrm{~nm}$ in Figure 5 suggests that the PFO aggregation is much higher in the blend films than that of the ES fibers. In addition, the absorption band of ES nanofibers is broadened as the PFO ratio is enhanced, which is due to a more inhomogeneous environment, as reported in other ES blend fibers. ${ }^{11 \mathrm{a}, 12 \mathrm{~b}}$

Parts a and $\mathrm{b}$ of Figure 6 show the photoluminescence spectra of the PFO/PMMA ES fibers and their corresponding spincoated blend films excited at $380 \mathrm{~nm}$. The corresponding emission maxima $\left(\lambda^{\mathrm{PL}}{ }_{\max }\right)$ and luminescence efficiency are summarized in Table 1 . At the low PFO blend ratios of $1-10$ wt $\%$, two emission peaks at 421 and $442 \mathrm{~nm}$ are observed in Figure $6 \mathrm{a}$ but additional emission peaks at $446 \mathrm{~nm}$ and at 466 and $501 \mathrm{~nm}$ are observed for 30 and 50 wt \% PFO fibers, respectively. For the case of spin-coated films, three emission peaks with maxima around 438-441, 463-469, and 495-500 $\mathrm{nm}$ are observed at all PFO blend compositions. Note that the PL emission maxima of PFO films at 436, 463, and $494 \mathrm{~nm}$ are due to different vibronic structures. ${ }^{7 a}$ The blue-shifted emission bands in the ES nanofibers in comparison with those of spin-coated films clearly indicates a the weaker interaction in the ES fibers, which could result from the small aggregation domain. Besides, The 50 wt \% PFO blend film shows a clear emission band at $533 \mathrm{~nm}$, which is suggested to be aggregate/ excimer emission ${ }^{15}$ although the keto defect may also lead to such emission band. ${ }^{6 b}$

Table 1 shows the internal quantum efficiency excited by $380 \mathrm{~nm}$ of PFO/PMMA fibers and their corresponding films. The quantum efficiency of the ES fibers and films decreases from 75.5 to $44.6 \%$ and 58.2 to $41.1 \%$, respectively, as the PFO blend ratio is increased from 1 to $50 \mathrm{wt} \%$. The trend between the quantum efficiency and the blend ratio could be explained by the enhanced PFO aggregation at the high PFO blend ratio since the aggregation/excimer generally reduces the fluorescence efficiency. ${ }^{15}$ The higher quantum efficiency of the ES fiber than that of the corresponding film on the same blend ratio indicates the lower PFO aggregation of the former.

Parts a-d of Figure 7 shows the laser confocal microscope images of ES nanofibers from 5 wt \% PFO/PMMA, PFQ/ PMMA, PFBT/PMMA, and PFTP/PMMA blends, respectively, which have the colors of blue, green, yellow, and red. It suggests that full color ES fibers could be produced from the PF derivative/PMMA blend. The fiber diameters of the above four blend systems are in the range of 250-750 nm, as shown in the FE-SEM images of the inset figure. The similar diameter of the above ES fibers is because $95 \%$ of the blend is PMMA and its higher molecular weight than the studied polyfluorene derivative. Parts a and b of Figure 8 show the optical absorption and photoluminescence spectra of the studied four blend ES fibers. The longest optical absorption maxima of the PFQ/ PMMA, PFBT/PMMA, and PFTP/PMMA ES fibers are observed at 406, 458, $531 \mathrm{~nm}$ while the emission maxima are at $483,539,(628,658)$, respectively. Note that the absorption
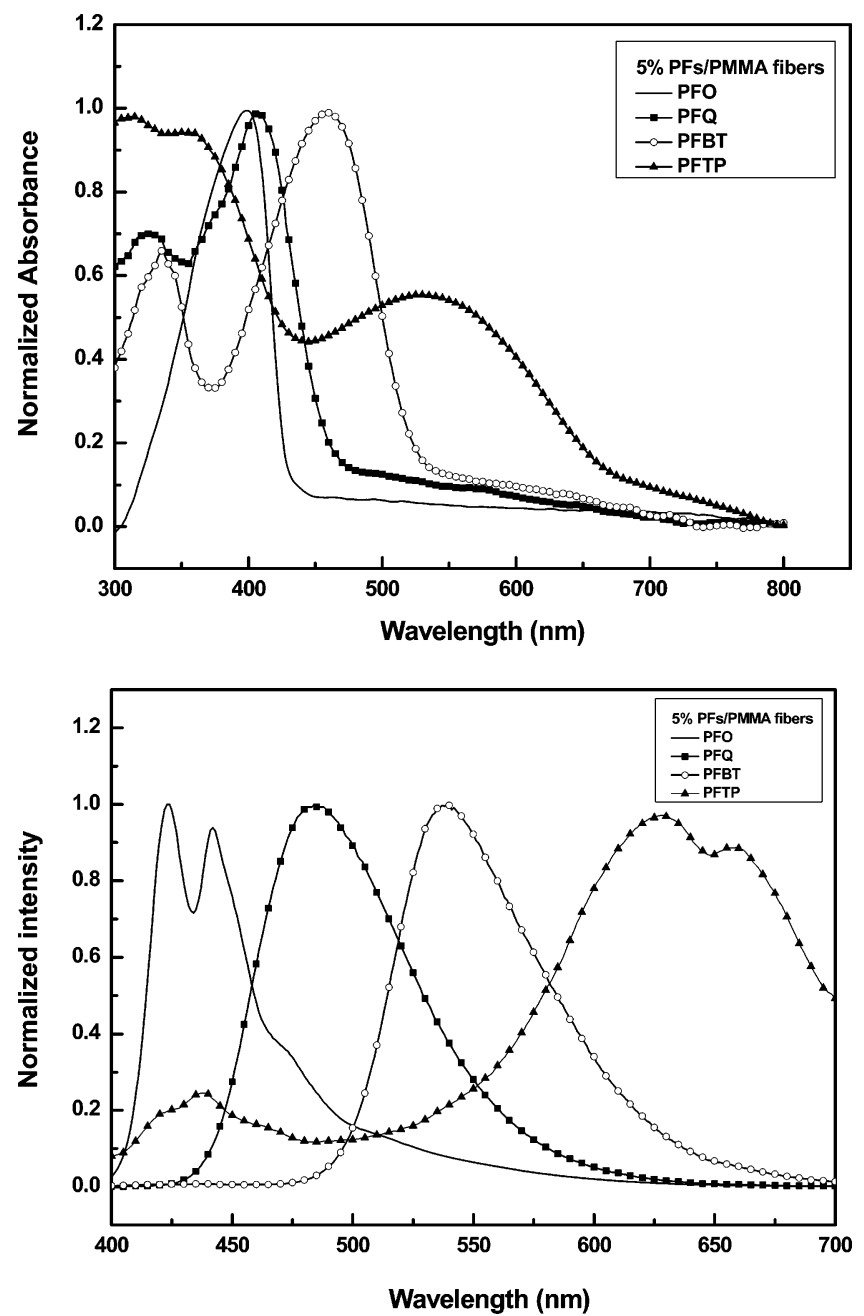

Figure 8. (a) Optical absorption and (b) photoluminescence emission spectra of 5 wt \% polyfluorene derivative /PMMA blend ES nanofibers.

maxima of the parent polymers, PFQ, PFBT, and PFTP are 416, 470 , and $578 \mathrm{~nm}$, respectively, while those of emission are 493, 540 , and $674 \mathrm{~nm}$, respectively. ${ }^{6 \mathrm{~d}}$ The above absorption maxima of the ES fibers are blue-shifted from those of thin films, as shown in Table 1, which could be due to the different aggregation size and interchain interaction. However, the comparison on the emission maxima between the ES fibers and films does not show a clear trend. The PL quantum efficiencies of the PFQ/PMMA, PFBT/PMMA, and PFTP/PMMA ES fibers are $33.3,24.5$, and $3.9 \%$, which are higher than those of the corresponding films with $11.2,15.4$, and $3.6 \%$, respectively. The aggregation degree between the ES fibers and the spincoated films probably results in the difference on the luminescence efficiency.

The present study suggests that high luminescence ES fibers with various emission colors could be produced by the polyfluorene derivative/PMMA blends. Such fibers could be potentially used for sensory device applications.

\section{Conclusions}

In this study, light-emitting electrospun nanofibers with the diameters of 250-750 $\mathrm{nm}$ were successfully prepared through the polyfluorene derivative/PMMA blends using a singlecapillary spinneret. The TEM results showed that uncontinuous fiber-like structure was obtained at the low PFO/PMMA blend ratio but became core-shell structure at a high $\mathrm{PFO}$ blend ratio. Besides, porous surface structure on the PFO/PMMA blend 
fibers was observed in the SEM images due to the rapid evaporation of the chloroform solvent. The polyfluorene aggregation in the ES fibers is much smaller than that in the spincoated films due to the geometrical confinement of the ES process and results in higher luminescence efficiency. Uniform ES fibers produced from the binary blends of PFO/PMMA, PFQ/PMMA, PFBT/PMMA, and PFTP/PMMA exhibited the luminescence colors of blue, green, yellow, and red, respectively. The present study demonstrates that full color light-emitting ES nanofibers could be produced from the binary blends of polyfluorene/ PMMA.

Acknowledgment. The financial support from the National Science Council of Taiwan, Ministry of Education, and Ministry of Economics Affairs is highly appreciated.

Supporting Information Available: Figures showing SEM images of PFO/PMMA blend ES fibers, laser confocal microscopy of PFO/PMMA ES fibers and films, and FE-SEM images of PFQ/ PMMA, PFBT/PMMA, and PFTP/PMMA ES fibers. This material is available free of charge via the Internet at http://pubs.acs.org.

\section{References and Notes}

(1) See Special Issue on Organic Electronics: Chem. Mater. 2004, 16, 4381-4846.

(2) (a) Burroughes, J. H.; Bradley, D. D. C.; Brown, A. R.; Marks, R. N.; Mackay, K.; Friend, R. H.; Burns, P. L.; Holmes, A. B. Nature (London) 1990, 347, 539; (b) Kraft, A.; Grimsdale, A. C.; Holmes, A. B. Angew. Chem., Int. Ed. 1998, 37, 402.

(3) (a) Chua, L. L.; Zaumseil, J.; Chang, J. F.; Ou, E. C.-W.; Ho, P. K.H.; Sirringhaus, H.; Friend, R. H. Nature (London) 2005, 434, 194. (c) Lin, M. M.; Bao, Z. Chem. Mater. 2004, 16, 4824.

(4) (a) Yu, G.; Gao, J.; Hummelen, J. C.; Wudl., F.; Heeger, A. J. Science 1995, 270, 1789; (b) Scharber, M. C.; Muhlbacher, D.; Koppe, M.; Denk, P.; Waldauf, C.; Heeger, A. J.; Brabec, C. J. Adv. Mater. 2006, 18, 789. (c) Li, G.; Shrotriya, V.; Huang, J.; Yao, Y.; Moriarty, T.; Emery, K.; Yang, Y. Nat. Mater. 2005, 4, 864.

(5) (a) Grell, M.; Bradley, D. D. C.; Inbasekaran, M.; Woo, E. P. Adv. Mater. 1997, 9, 798. (b) Neher, D. Macromol. Rapid Commun. 2001, 22, 1365. (c) Schref, U.; List, E. J. W. Adv. Mater. 2002, 14, 477.

(6) (a) Peng, Q.; Peng, J. B.; Kang, E. T.; Neoh, K. G.; Cao, Y. Macromolecules 2005, 38, 7292; (b) Lin, W. J.; Chen, W. C.; Wu, W. C.: Niu, Y. H.; Jen, A. K. Y. Macromolecules 2004, 37, 2335; (c) Wu, W. C.; Lee, W. Y.; Chen, W. C. Macromol. Chem. Phys. 2006, 207, 1131. (d) Wu, W. C.; Liu, C. L.; Chen, W. C. Polymer 2006, 47,
527. (e) Chuang, C. Y.; Shih, P. I.; Chieh, C. H.; Wu, F. I.; Shu, C. F. Macromolecules 2007, 40, 247.

(7) (a) Kulkarni, A. P.; Jenekhe, S. A. Macromolecules 2003, 36, 5285; (b) Iyengar, N. A.; Harrison, B.; Duran, R. S.; Schanze, K. S.; Reynolds, J. R. Macromolecules 2003, 36, 8978; (c) Ananthakrishnan, N.; Padmanaban, G.; Ramakrishnan, S.; Reynolds, J. R. Macromolecules 2005, 38, 7600; (d) Rathnayake, H. P.; Cirpan, A.; Lahti, P. M.; Karasz, F. E. Chem. Mater. 2006, 18, 560.

(8) (a) Li, D.; Xia, Y. Adv. Mater. 2004, 16, 1151. (b) Reneker, D. H.; Chun, I. Nanotechnology 1996, 7, 216. (c) Huang, Z. M.; Zhang, Y Z.; Kotaki, M.; Ramakrishna, S. Compos. Sci. Technol. 2003, 63, 2223. (d) Dersch, R.; Steinhart, M.; Boudriot, U.; Greiner, A.; Wendorff, J. H. Polym. Adv. Technol. 2005, 16, 276.

(9) (a) Fridrikh, S. V.; Yu, J. H.; Brenner, M. P.; Rutledge, G. C. Phys. Rev. Lett. 2003, 90, 144502-1. (b) Wang, C.; Hsu, C. H.; Lin, J. H. Macromolecules 2006, 39, 7662. (c) Kalra, V.; Kakad, P. A.; Mendez, S.; Ivannikov, T.; Kamperman, M.; Joo, Y. L. Macromolecules 2006, 39, 5453. (d) Ma, M.; Krikorian, V.; Yu, J. H.; Thomas, E. L.; Rutledge, G. C. Nano. Lett. 2006, 6, 2969. (e) Bazilevsky, A. V.; Yarin, A. L.; megaridis, C. M. Langmuir 2007, 23, 2311. (f) Choi, J. S.; Lee, S. W.; Leong, L.; Bae, S. H.; Min, B. C.; Youk, J. H.; Park, W. H. Int. J. Bio. Macromol. 2004, 34, 249. (g) Choktaweesap, N.; Arayanarakul, K.; Aht-Ong, D.; Meechaisue, C.; Supaphol, P. Polym. J. 2007, 39, 622 .

(10) (a) MacDiarmid, A. G.; Jones, W. E. Jr.; Llaguno, M. Synth. Met. 2001, 119, 27. (b) Dong, H.; Nyame, V.; MacDiarmid, A. G.; Jones, W. E. Jr. J. Polyms. Sci. Polym. Phys. 2004, 42, 3934. (c) Wei, M.; Lee, J.; Kang, B.; Mead, J. Macromol. Rapid Commun. 2005, 26, 1127. (d) Jang, S. Y.; Seshadri, V.; Khil, M. S.; Kumar, A.; Marquez, M.; Mather, P. T.; Sotzing, G. A. Adv. Mater. 2005, 17, 2177.

(11) (a) Madhugiri, S.; Dalton, A.; Balkus, K. J, Jr. J. Am. Chem. Soc. 2003, 125, 14531. (b) Wang, X.; Kim, Y. G.; Drew, C.; Ku, B.-C. Kumar, J.; Samuelson, L. A. Nano. Lett. 2004, 4, 331. (c) Wang, X.; Drew, C.; Lee, S.-H.; Senecat, K. J.; Kumar, J.; Samuelson, L. A. Nano Lett. 2002, 2, 1273. (d) Wutticharoenmongko, P.; Supaphol, P.; Srikhirin, T.; Kerdcharoen, T.; Osotchan, T. J. Polym. Sci., Polym. Phys. 2005, 43, 1881. (e) Xin, Y.; Huang, Z. H.; Yan, E. Y.; Zhang, W.; Zhao, Q. Appl. Phys. Lett. 2006, 89, 053101. (f) Kwak, G.; Fukao, S.; Fujiki, M.; Sakaguchi, T.; Masuda, T. Chem. Mater. 2006, 18, 5537. (g) Wang, Y.; Park, J. S.; Leech, J. P.; Miao, S.; Bunz, U. H. F. Macromolecules 2007, 40, 1843 .

(12) (a) Li, D.; Babel, A.; Jenekhe, S. A.; Xia, Y. Adv. Mater. 2004, 16, 2062. (b) Babel, A.; Li, D.; Xia, Y.; Jenekhe, S. A. Macromolecules 2005, 38, 4705. (c) Okuzaki, H.; Takahaski, T.; Miyajima, N.; Suzuki, Y.; Kuwabara, T. Macromlecules 2006, 39, 4276.

(13) Pedicine, A.; Farris, R. J. Polymer 2003, 44, 6857

(14) Lam, J. W. Y.; Tang, B. Z. Acc. Chem. Res. 2005, 38, 745.

(15) Jenekhe, S. A.; Osaheni, J. A. Science 1994, 265, 765. PFO has a strong interchain $\pi-\pi$ interaction and easily to form an aggregation domain, which results in the red-shift on the luminescence band Excimer is formed by interaction of an excited chromophore with an unexcited chromophore. Such an excited-state complex usually results in the red-shift of the luminescence band of conjugated polymers. 